This item is the archived peer-reviewed author-version of:

Pertussis vaccination in pregnancy : state of the art

\title{
Reference:
}

Leuridan Eke.- Pertussis vaccination in pregnancy : state of the art

Vaccine / International Society for Vaccines - ISSN 0264-410X - Oxford, Esevier sci Itd, 35:35A(2017), p. 4453-4456

Full text (Publisher's DOI): https://doi.org/10.1016/J.VACCINE.2017.03.061

To cite this reference: http://hdl.handle.net/10067/1454460151162165141 
2 Elke Leuridan, $\mathrm{MD}, \mathrm{PhD}^{*}$

3

$4 *$ Center for the Evaluation of Vaccinations, Vaccine \& Infectious Diseases Institute, Faculty of

5 Medicine and Health Sciences, University of Antwerp, Belgium

$6 \quad$ Universiteitsplein 1

72610 Wilrijk

$8 \quad$ Tel 003232652885

$9 \quad$ Email: elke.leuridan@uantwerpen.be

10

11 Highlights: Pertussis vaccination in pregnancy is safe, immunogenic and effective

12

13 Keywords: pertussis, vaccine, pregnancy, infants, maternal antibodies

14

15 Acknowledgement: All collaborators with the Centre for the Evaluation of Vaccination, in the past and ongoing studies on maternal immunization, in Belgium and abroad, are acknowledged for their valuable support and fruitful cooperation. EL is beneficiary of a postdoctoral mandate fellowship from the FWO (FWO 12D6114N).

19 
Part of protection against infectious diseases at birth, is provided by maternal antibodies transported via the placenta during pregnancy, and via lactation afterwards. These maternal antibodies wane during the first months of life: the interval between the loss of maternal protection and the onset of infant vaccine-induced protection should be as narrow as possible for all vaccine-preventable diseases $[1,2]$.

In regard to pertussis, neonates are most prone to severe disease and death. Despite high coverages in globally introduced vaccination programs, there are increasingly outbreaks of pertussis. Cause of the recent resurgence in high income countries, is multi-factorial [3], including the switch from whole cell Pertussis (wP) to acellular Pertussis (aP) vaccines with consequences on Thelper-1 (Th1)-Th17 versus Th2 cellular immune responses, a faster waning of immunity after aP vaccination in paediatric, adolescent and adult non-pregnant populations, reduced impact on infection and transmission by the aP vaccines, etc. The recent resurgence in wP using countries relies on low coverage and possibly poor vaccine quality [4]. In either situation, neonates are most prone to severe disease and death. Better vaccines, inducing longer protection, would be an asset in the combat of pertussis disease.

During recent epidemics, national advisory bodies (e.g. United States of America (US) (2011)[5], United Kingdom (UK) (2012)[6], Belgium (2013)[7]) had no other option than to recommend pertussis vaccination with a tetanus, diphtheria and acellular pertussis (Tdap) vaccine during pregnancy, to offer passive protection from immediately at birth, thus closing the neonatal susceptibility gap. There were, however, major gaps in the scientific knowledge at the moment of recommendation: on safety, immunogenicity, interference of maternal antibodies with aP or WP infant vaccine responses, breast milk composition, etc. The present review offers an overview on new insights and still existing knowledge gaps in this quickly evolving research domain.

\section{Safety of pertussis vaccination during pregnancy}


Maternal and obstetrical safety, as well as safety for the foetus and after birth during infancy, have to be considered and monitored when immunizing during pregnancy. The first large prospective study on safety of Tdap administration in pregnant women, was performed after implementation of the recommendation in the UK [8]. A number of outcomes were monitored, showing no increased risk of stillbirth, maternal or neonatal death, (pre-) eclampsia, and other predefined conditions. US data [9] assessed also the risk of infants born small for gestational age (SGA), prematurity, hypertensive disorders and chorioamnionitis after Tdap during pregnancy, in a large cohort of 123,494 women. No increased risk was found, except for a small relative risk (RR) increase of 1.19 for chorioamnionitis. Chorioamnionitis data were therefore further investigated in the VAERS database (Vaccine Adverse Event Reporting System, Centers for Disease Control and prevention (CDC)) and the relationship with Tdap administration during pregnancy could not be confirmed [10]. Very recently, the GAIA (Global Alignment of Immunisation Safety Assessment in Pregnancy) consortium, supported by the Brighton Collaboration, offers a detailed frame for collection and analysis of safety data gathered during pregnancy vaccine trials [11].

In summary, all available safety data on Tdap during pregnancy are certainly reassuring [12], yet the importance of comprehension of background incidence rates and the need for surveillance of adverse events, should be stressed $[12,13]$; also in low and middle income countries (LMIC) where there is often a higher burden of concomitant diseases and adverse pregnancy outcomes [14, 15]. Since Tdap is recommended to be administered during every consecutive pregnancy, in order to have as high a titer of maternal antibodies as possible, the safety of repeat tetanus containing vaccines was recently reviewed and confirmed to be of no harm [16].

\section{Immunogenicity of vaccination during pregnancy}

The immune system during pregnancy alters in function of the tolerance of the foetus, but humoral immune responses are not different during pregnancy as compared to a non- pregnant immune status [17]. For adults, only combination vaccines are available against pertussis (aP), containing also 
tetanus, diphtheria, and sometimes polio. Depending on the manufacturer, composition differs in the number and amount of the inactivated pertussis components. AP vaccines induce high concentrations of antibodies during pregnancy [18-23] and similar humoral responses were reported in pregnant as in non-pregnant women [24], yet the stimulation of vaccine specific Th1 type cellular immune responses seems to be transient and impaired during pregnancy. The importance of that finding needs more in depth research, in view of duration of protection from disease.

There is no correlate of protection for pertussis, but higher antibody concentrations against pertussis toxin (PT) and to a lesser extent pertactin (Prn), are related to better protection [25]. Until now, few data on the functionality, or neutralizing capacity, of the maternal antibodies induced during pregnancy, have been published [26].

\section{The role of maternal antibodies in protecting young infants from disease}

Several studies confirmed the beneficial effect of maternal prenatal vaccination on the antibody titer in cord blood with endured higher concentrations of antibodies in neonates until primary vaccination is started $[20,22,23,27]$. The maternal IgG antibodies are transported in utero by the placental neonatal $\mathrm{Fc}$ receptor ( $\mathrm{FCRn}$ ), and the transport is most efficient during the last trimester of gestation [17]. Recent Swiss data indicate however, that a longer exposure to higher concentrations of maternally circulating antibodies is beneficial for the concentration of maternal antibodies in cord blood (rather second trimester than third trimester vaccination), and hence protection during the first months of life [28]. A small study showed that even a pre-pregnancy Tdap booster also induced higher antibody levels in cord blood compared to a control group [29]. The peak response to Tdap vaccination in adult women occurs at 2 weeks postvaccination [30] and antibodies decline by half at 12 months post-vaccination in the women [24].

Since transplacental transport is more efficient near term delivery, preterm infants receive significantly less antibodies [31], and they might benefit from earlier vaccination during pregnancy despite the impaired transplacental transport at the beginning of the third trimester. The optimal 
gestational age for Tdap vaccination is still subject for debate [28], but effectiveness data are better when there is an interval of at least 4 weeks between vaccination and delivery [21, 28].

In terms of effectiveness, historical data on maternal vaccination with wP vaccines have shown effectiveness of the strategy to protect young infants from disease [32]. Recently, baboon challenge studies confirm the protective effect of maternal aP vaccination during pregnancy [33, 34], however, offspring was protected from disease, yet not from infection. UK experiences indicate that there were significantly less deaths after the recommendation was put in place $[35,36]$, with a sustained effect 3 years after implementation. In addition, the number of hospitalisations of young infants for pertussis disease after the recommendation was made, reduced significantly, with a more pronounced effect whenever prenatal vaccination was performed with larger interval before delivery. Overall mortality decreased also in Argentina after introduction of the maternal vaccination strategy, with highest reduction rate among the very young [37, 38]. Winter et al [39] report effectiveness of the strategy on the severity of the pertussis cases among young infants whose mothers were vaccinated: they has significantly lower risk of hospitalisation and intensive care unit admission and shorter hospital stay.

\section{$\underline{\text { Interference by maternal antibodies on infant immune responses }}$}

In the presence of high concentrations of maternal antibodies (naturally induced after infection), blunting of the infant responses to wP vaccines has been described in the past [40-42]. At present, studies in UK [43], Belgium [23] and Vietnam [22] report significant blunting of infant responses on aP antigens in infant vaccines, whenever their mother was vaccinated during pregnancy. In the UK study, humoral response to some serotypes in the pneumococcal infant vaccination was also significantly lower, due to the use of Diphtheria CRM 197 conjugation in both vaccines during pregnancy (Tdap) and infancy (pneumococcal conjugate vaccines). In Belgium, even the post-booster immune response to pertussis toxin was still affected by the maternal immunisation [44]. The clinical repercussion of this blunting remains uncertain. We need more detailed data on infant responses in 
the presence of maternal antibodies, to vaccines from several manufacturers (different composition of antigens), with different infant vaccine schedules and distinct starting ages, intervals, and number of doses.

Knowledge on the functionality of the infant antibodies, induced in the presence of maternal antibodies, is lacking as well as insight in the cellular immune responses of young infants after immunisation, in the presence of high concentrations of maternal antibodies. Several initiatives are ongoing to unravel these questions. Delaying the primary infant vaccination to avoid interference, would need high coverages of the recommended strategy in order not to have a longer susceptibility gap in infants from unvaccinated women.

Interference should also be kept in mind, when extending the recommendations to LMIC. Tetanus vaccination is recommended during pregnancy within the EPI schedule, with a global coverage of over 75\% in 2012 [45]. This strategy is well accepted, offering a platform to possibly add pertussis vaccine, since high burden of pertussis disease is estimated in LMIC [45], despite underreporting, under-diagnosis and a lack of technical possibilities for confirmation of cases. Given the data on blunting effects by maternal antibodies, the influence of high concentrations of aP induced maternal antibodies on infant vaccine responses to a WP vaccines, should first be investigated.

\section{Adherence to recommendations}

One crucial factor in this entire story, is the acceptance of the strategy by the health care professional (HCP) taking care of pregnant women, and the acceptance by the target group. National coverage data differ in countries where the recommendation has been put in place: UK coverage went up to over $80 \%$ at the start of the implementation in 2012 and was 56.4\% in 2014 [46]; in Flanders, Belgium, the coverage was $64 \%$ in 2014 [47] , and in Argentina the coverage was $56.9 \%$ in 2014 [38]. A review on the influencing factors for vaccine acceptance during pregnancy [48] described that the main barriers for the women related to vaccine safety, belief that the vaccine is not needed or effective, lack of recommendation by HCP, low knowledge about vaccines, access 
152 issues, cost, conflicting advice. The HCP barriers related to inadequate training and inadequate

153 workload. We confirmed in Belgium a few of these hurdles and identified specific at risk groups for

154 not receiving the vaccine, mainly in a sociological context, as did others: women with migration

155 background [49-52], lower maternal educational level [49], maternal unemployment, provider's

156 attitude (not offered by HCP or discouraged by HCP) [47].

157

158

159 
Conclusion

Pertussis immunisation during pregnancy has been recommended in several high income countries, as it is the only possible means, with the currently available vaccines, to protect young infants from (severe) pertussis disease. The strategy is safe and effective, based on the currently available data. There are indications of a blunting effect by the vaccine induced maternal antibodies, on the infant immune responses to aP vaccines, yet the clinical meaning is unclear. Despite research on other maternal vaccination possibilities, like flu and tetanus, many gaps remain in our basic knowledge, included in Table 1.

Table 1: existing evidence (Including indications of knowledge, that might need more evidence) and knowledge gaps regarding Tdap vaccination in pregnancy.

Research opportunities are plenty, in this quickly evolving field.

The future looks promising: the gained knowledge on pertussis and flu, will and is already used to combat other diseases e.g. RSV infection in young infants, GBS infection in neonates and congenital CMV disease, for which maternal immunisation will certainly make a difference in reducing infant disease burden.

\section{References}

[1] Leuridan E, Hens $N$, Hutse V, leven $M$, Aerts $M$, Van Damme P. Early waning of maternal measles antibodies in era of measles elimination: Iongitudinal study. British Medical Journal. 2010;340:c1626. [2] Leuridan E, Hens N, Hutse V, Aerts M, Van Damme P. Kinetics of maternal antibodies against rubella and varicella in infants. Vaccine. 2011;29:2222-6.

[3] Saadatian-Elahi M, Plotkin S, Mills KH, Halperin SA, Mclntyre PB, Picot V, et al. Pertussis: Biology, epidemiology and prevention. Vaccine. 2016;34:5819-26.

[4] Cherry JD, Paddock CD. Pathogenesis and histopathology of pertussis: implications for immunization. Expert review of vaccines. 2014;13:1115-23.

[5] ACIP. ACIP Provisional Recommendations for Pregnant Women on Use of Tetanus Toxoid, Reduced Diphtheria Toxoid and Acellular Pertussis Vaccine (Tdap). 2011. 
[6] Department of Health E. Whooping cough vaccination programme for pregnant women: extension to 2014. 2013.

193 [7] Hoge Gezondheidsraad B. Pertussis vaccination. 2013.

194 [8] Donegan K, King B, Bryan P. Safety of pertussis vaccination in pregnant women in UK:

195 observational study. BMJ. 2014;349:g4219.

[9] Kharbanda EO, Vazquez-Benitez G, Lipkind H, Naleway AL, Klein NP, Cheetham TC, et al. Receipt of pertussis vaccine during pregnancy across 7 Vaccine Safety Datalink sites. Preventive medicine.

198 2014;67:316-9.

199 [10] Datwani H, Moro PL, Harrington T, Broder KR. Chorioamnionitis following vaccination in the Vaccine Adverse Event Reporting System. Vaccine. 2015;33:3110-3.

[11] Jones CE, Munoz FM, Spiegel HM, Heininger U, Zuber PL, Edwards KM, et al. Guideline for collection, analysis and presentation of safety data in clinical trials of vaccines in pregnant women. Vaccine. 2016;34:5998-6006.

[12] Keller-Stanislawski B, Englund JA, Kang G, Mangtani P, Neuzil K, Nohynek H, et al. Safety of immunization during pregnancy: a review of the evidence of selected inactivated and live attenuated vaccines. Vaccine. 2014;32:7057-64.

[13] Moro PL, McNeil MM, Sukumaran L, Broder KR. The Centers for Disease Control and Prevention's public health response to monitoring Tdap safety in pregnant women in the United States. Human vaccines \& immunotherapeutics. 2015;11:2872-9.

[14] Cutland CL, Cunnington M, Olugbosi M, Jones SA, Hugo A, Maharaj K, et al. Lessons learnt from enrolment and follow up of pregnant women and their infants in clinical trials in South Africa, a lowmiddle income country. Vaccine. 2015;33:6406-12.

[15] Fulton TR, Narayanan D, Bonhoeffer J, Ortiz JR, Lambach P, Omer SB. A systematic review of adverse events following immunization during pregnancy and the newborn period. Vaccine. 2015;33:6453-65.

[16] Sukumaran L, McCarthy NL, Kharbanda EO, McNeil MM, Naleway AL, Klein NP, et al. Association of Tdap Vaccination With Acute Events and Adverse Birth Outcomes Among Pregnant Women With Prior Tetanus-Containing Immunizations. JAMA. 2015;314:1581-7.

[17] Faucette AN, Unger BL, Gonik B, Chen K. Maternal vaccination: moving the science forward. Human reproduction update. 2015;21:119-35.

[18] Gall SA, Myers J, Pichichero M. Maternal immunization with tetanus-diphtheria-pertussis vaccine: effect on maternal and neonatal serum antibody levels. American journal of obstetrics and gynecology. 2011;204:334 e1-5.

[19] Healy CM, Munoz FM, Rench MA, Halasa NB, Edwards KM, Baker CJ. Prevalence of pertussis antibodies in maternal delivery, cord, and infant serum. Journal of Infectious Diseases. 2004;190:33540.

[20] Munoz FM, Bond NH, Maccato M, Pinell P, Hammill HA, Swamy GK, et al. Safety and immunogenicity of tetanus diphtheria and acellular pertussis (Tdap) immunization during pregnancy in mothers and infants: a randomized clinical trial. JAMA. 2014;311:1760-9.

[21] Abu Raya B, Srugo I, Kessel A, Peterman M, Bader D, Gonen R, et al. The effect of timing of maternal tetanus, diphtheria, and acellular pertussis (Tdap) immunization during pregnancy on newborn pertussis antibody levels - a prospective study. Vaccine. 2014;32:5787-93.

[22] Hoang HT, Leuridan E, Maertens K, Nguyen TD, Hens N, Vu NH, et al. Pertussis vaccination during pregnancy in Vietnam: Results of a randomized controlled trial Vaccine. 2016;34:151-9.

[23] Maertens K, Cabore RN, Huygen K, Hens N, Van Damme P, Leuridan E. Pertussis vaccination during pregnancy in Belgium: Results of a prospective controlled cohort study. Vaccine. 2016;34:14250 .

[24] Huygen K, Cabore RN, Maertens K, Van Damme P, Leuridan E. Humoral and cell mediated immune responses to a pertussis containing vaccine in pregnant and nonpregnant women. Vaccine. 2015;33:4117_23. 
[25] Taranger J, Trollfors B, Lagergard T, Sundh V, Bryla DA, Schneerson R, et al. Correlation between pertussis toxin IgG antibodies in postvaccination sera and subsequent protection against pertussis. The Journal of infectious diseases. 2000;181:1010-3. [26] Raya BA, Bamberger E, Almog M, Peri R, Srugo I, Kessel A. Immunization of pregnant women against pertussis: The effect of timing on antibody avidity. Vaccine. 2015;33:1948-52. and pertussis vaccine during pregnancy: transfer of maternal pertussis antibodies to the newborn. Vaccine. 2015;33:1056-62.

[28] Eberhardt CS, Blanchard-Rohner G, Lemaitre B, Boukrid M, Combescure C, Othenin-Girard V, et al. Maternal Immunization Earlier in Pregnancy Maximizes Antibody Transfer and Expected Infant Seropositivity Against Pertussis. Clinical infectious diseases : an official publication of the Infectious Diseases Society of America. 2016;62:829-36.

[29] Leuridan E, Hens N, Peeters N, de Witte L, Van der Meeren O, Van Damme P. Effect of a prepregnancy pertussis booster dose on maternal antibody titers in young infants. The Pediatric infectious disease journal. 2011;30:608-10.

[30] Halperin BA, Morris A, Mackinnon-Cameron D, Mutch J, Langley JM, McNeil SA, et al. Kinetics of the antibody response to tetanus-diphtheria-acellular pertussis vaccine in women of childbearing age and postpartum women. Clinical infectious diseases : an official publication of the Infectious Diseases Society of America. 2011;53:885-92.

[31] Ercan TE, Sonmez C, Vural M, Erginoz E, Torunoglu MA, Perk Y. Seroprevalance of pertussis antibodies in maternal and cord blood of preterm and term infants. Vaccine. 2013;31:4172-6.

[32] Cohen P, Scadron SJ. The effects of active immunization of the mother upon the offspring. The Journal of pediatrics. 1946;29:609-19.

[33] Warfel JM, Zimmerman LI, Merkel TJ. Acellular pertussis vaccines protect against disease but fail to prevent infection and transmission in a nonhuman primate model. Proc Natl Acad Sci U S A. 2014;111:787-92.

[34] Warfel JM, Papin JF, Wolf RF, Zimmerman LI, Merkel TJ. Maternal and neonatal vaccination protects newborn baboons from pertussis infection. The Journal of infectious diseases. 2014;210:604-10.

[35] Amirthalingam G, Letley L, Campbell H, Green D, Yarwood J, Ramsay M. Lessons learnt from the implementation of maternal immunization programs in England. Human vaccines \& immunotherapeutics. 2016;12:2934-9.

[36] Amirthalingam G, Campbell H, Ribeiro S, Fry NK, Ramsay M, Miller E, et al. Sustained Effectiveness of the Maternal Pertussis Immunization Program in England 3 Years Following Introduction. Clinical infectious diseases : an official publication of the Infectious Diseases Society of America. 2016;63:S236-S43.

[37] Vizzotti C, Juarez MV, Bergel E, Romanin V, Califano G, Sagradini S, et al. Impact of a maternal immunization program against pertussis in a developing country. Vaccine. 2016;34:6223-8.

[38] Vizzotti C, Neyro S, Katz N, Juarez MV, Perez Carrega ME, Aquino A, et al. Maternal immunization in Argentina: A storyline from the prospective of a middle income country. Vaccine. 2015;33:6413-9. [39] Winter K, Cherry JD, Harriman K. Effectiveness of Prenatal Tetanus, Diphtheria, and Acellular Pertussis Vaccination on Pertussis Severity in Infants. Clinical infectious diseases : an official publication of the Infectious Diseases Society of America. 2017;64:9-14.

[40] Sako W TW, Witt DB, et al. Early immunization against pertussis with alum precipitated vaccine. Jama. 1945;127:379-83.

[41] Provenzano RW WL, Sullivan CL. . Immunization and antibody response in the newborn infant. The New England journal of medicine. 1965;273:959-65.

[42] Englund JA, Anderson EL, Reed GF, Decker MD, Edwards KM, Pichichero ME, et al. The effect of maternal antibody on the serologic response and the incidence of adverse reactions after primary immunization with acellular and whole-cell pertussis vaccines combined with diphtheria and tetanus toxoids. Pediatrics. 1995;96:580-4. 
[43] Ladhani SN, Andrews NJ, Southern J, Jones CE, Amirthalingam G, Waight PA, et al. Antibody responses after primary immunization in infants born to women receiving a pertussis-containing vaccine during pregnancy: single arm observational study with a historical comparator. Clinical infectious diseases : an official publication of the Infectious Diseases Society of America. 2015;61:1637-44.

[44] Maertens K, Cabore RN, Huygen K, Vermeiren S, Hens N, Van Damme P, et al. Pertussis vaccination during pregnancy in Belgium: Follow-up of infants until 1 month after the fourth infant pertussis vaccination at 15 months of age. Vaccine. 2016;34:3613_9. [45] Tan T, Dalby T, Forsyth K, Halperin SA, Heininger U, Hozbor D, et al. Pertussis Across the Globe:

\section{Conflict of interest statement}

Authors do not have a commercial or other association that might pose a conflict of interest (e.g., 